\title{
Topology Preserving Warping of Binary Images: Application to Atlas-Based Skull Segmentation
}

\author{
Sylvain Faisan ${ }^{1}$, Nicolas Passat ${ }^{1}$, Vincent Noblet $^{1}$, Renée Chabrier ${ }^{2}$, \\ and Christophe Meyer ${ }^{3}$ \\ ${ }^{1}$ LSIIT - UMR CNRS 7005, Strasbourg I University, France \\ ${ }^{2}$ LINC - UMR CNRS 7191, Strasbourg I University, France \\ ${ }^{3}$ University Hospital of Besançon, France
}

\begin{abstract}
Lots of works have been recently carried out in the field of non-rigid registration to ensure the estimation of one-to-one mappings. However, warping a binary image with such transformations may alter its discrete topological properties if common resampling strategies are considered. This paper proposes an original method for warping a binary image according to some continuous and bijective mapping, while preserving its discrete topological properties. Results obtained in the context of atlas-based segmentation highlight the interest of the approach. Indeed, the method has been successfully applied to the segmentation of skull structures from a database of 15 CT-scans, providing both geometrically and topologically satisfactory results.
\end{abstract}

\section{Introduction}

Image warping is the process of applying some geometric transformation to an image. Given an image $M$ and a continuous deformation field $h$, the goal is to compute the warped image $S$, so that for each voxel $v, S(v)=M(h(v))$. Since $h(v)$ does not necessarily correspond with grid point, some interpolation techniques are required to evaluate $M(h(v))$.

Although several image interpolation techniques (linear, cubic) [1 have been proposed for grey-level images, no specific attention has been paid to the case of binary data. Common interpolation techniques, except the nearest neighbour interpolation, do not guarantee the resampled image $S$ to remain a binary image. To circumvent this limitation, it is possible to use a thresholding as post-processing of interpolation to get a binary image. Unfortunately, warping a discrete image according to a continuous and bijective (i.e. topology-preserving) deformation field with these common interpolation techniques may fail in preserving its discrete topological properties. Quite surprisingly, many works have been devoted to develop registration methods providing deformation fields which preserve the continuous topology, while the topology preservation of discrete objects deformed by such fields has not yet been considered. Based on these considerations, we propose an algorithm for warping a binary image according to a topology-preserving deformation field without altering its discrete topology.

D. Metaxas et al. (Eds.): MICCAI 2008, Part I, LNCS 5241, pp. $211 \sqrt{218} 2008$.

(C) Springer-Verlag Berlin Heidelberg 2008 
The proposed approach is inspired from concepts generally considered in the context of segmentation, where topology preservation is a crucial issue. The segmentation methods dealing with this constraint are often based on the concept of 3-D simple points 2 (i.e. points whose addition or removal from a binary object does not alter its topology), which can be locally characterised in constant time, leading to fast algorithms. The basic idea of the proposed method is to modify the initial image in a homotopy-preserving fashion by adding and removing simple points until converging to a solution that is as close as possible to the continuous warped image.

The paper is organised as follows. In Section 2 the proposed method is described. In Section 3. results in the context of atlas-based segmentation are presented. Conclusions and perspectives are provided in Section 4

\section{Method}

The method of warping a binary image $M$ according to a continuous and bijective deformation field $h$ can be stated as the following constrained optimisation problem:

$$
\hat{S}=\arg \min _{S \sim M} d(S, M, h),
$$

where $S$ is a binary image constrained to be topologically equivalent to $M$ and $d(S, M, h)$ a distance between $S$ and the continuous warped image $M(h)$. This paper presents a method to tackle this problem. We first introduce in Sec. 2.1 the distance $d(S, M, h)$. Then, we explain in Sec. 2.2 how to constrain $S$ to be topologically equivalent to $M$ during the optimisation process. Finally, the optimisation strategy is detailed in Sec. 2.3. A global overview of the method is given in Alg. 1.

\subsection{Cost Function}

Since $M$ and $S$ are constrained to have the same topology, there is a one-toone relation between connected components (CCs) of $M$ and the ones of $S$. These CCs can be background CCs (BCCs) or object CCs (OCCs), each CC corresponding to a distinct label. We define $\mathcal{N}(v, S, M)$ as the CC in $M$ which corresponds to the $\mathrm{CC}$ in $S$ that encloses voxel $v$. The distance $d(S, M, h)$ between $S$ and the continuous warped image $M(h)$ is considered hereafter as the cost function, and is computed as follows:

$$
d(S, M, h)=\sum_{v \in S} \rho(v, S, M, h), \text { with } \rho(v, S, M, h)=\min _{v^{\prime} \in \mathcal{N}(v, S, M)}\left\|v^{\prime}-h(v)\right\|,
$$

where $\rho(v, S, M, h)$ is the distance between $h(v)$ and the CC of $M$ which is associated to the CC that encloses $v$ in $S$. To clarify the idea, the computation of the cost function is illustrated in a 2-D case in Fig. 11 $\rho(v, S, M, h)$ can be efficiently evaluated by computing the chamfer distance map of the $\mathrm{CC}$ of $M$ (the one associated to the CC that encloses $v$ in $S$ ) and by evaluating its value at position $h(v)$. Notice that the chamfer distance map can be computed one time for each CC. 

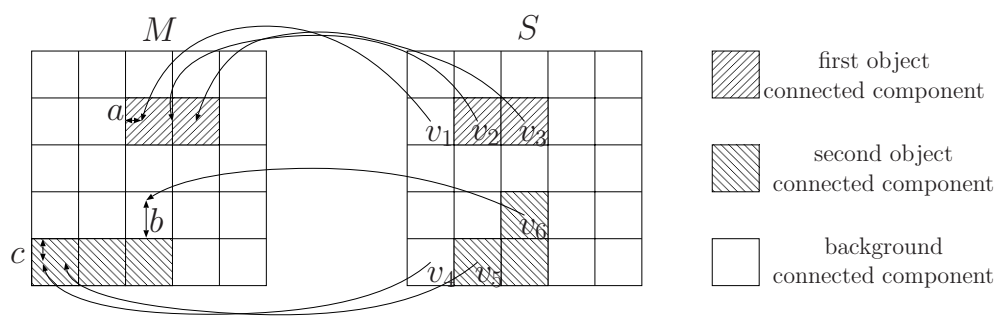

Fig. 1. Illustration of the computation of $\rho(v, S, M, h)$ for 6 pixels. $M$ is composed of two OCCs and of one BCC. $\rho(v, S, M, h)$ is equal to 0 for $v_{2}, v_{3}$, and $v_{5}$ since $h\left(v_{2}\right)$, $h\left(v_{3}\right)$, and $h\left(v_{5}\right)$ belong to the same $\mathrm{CC}$ as $v_{2}, v_{3}$, and $v_{5}$, respectively. However, $v_{6}$ belongs to the second $\mathrm{OCC}$ whereas $h\left(v_{6}\right)$ belongs to the BCC so that $\rho\left(v_{6}, S, M, h\right)$ is equal to $b$, namely, the distance between $h\left(v_{6}\right)$ and the second OCC (in $M$ ). In the same way, $\rho\left(v_{1}, S, M, h\right)=a$ and $\rho\left(v_{4}, S, M, h\right)=c$.

\subsection{Topology Handling}

At the beginning of the method, $S$ is initialized to $M$ and is then modified by iterative removal/addition of simple points. The label of a simple point is changed if it decreases the cost function. When changing the label of $v$, it is associated unambiguously to a unique CC, since it is a simple point. To determine this CC, two images representing the labels of $S$ are used. They are updated during the whole process with $S$.

The removal/addition of simple points can be not appropriated when a voxel has to be "translated". The translation can be interpreted in terms of an addition (resp. a removal) followed by a removal (resp. an addition) of simple points. However, the cost function is estimated after each label modification. Consequently, the first modification may increase the cost function, leading to refuse this operation, whereas both modifications may decrease the cost function. That is why the concept of topology-preserving translation is defined. This notion is interpreted here as the simultaneous modification of the status of a couple $\left(v, v^{\prime}\right)$ of adjacent voxels such that $S(v)=1-S\left(v^{\prime}\right)$. To guarantee topology preservation, it is sufficient to check that $v$ (resp. $v^{\prime}$ ) is simple for $S$ and $v^{\prime}$ (resp. $v$ ) is simple in $S^{\prime}$ obtained from $S$ after the modification of $v$ (resp. $\left.v^{\prime}\right)$. The translation at voxel $v$ is performed if it actually reduces the cost function. If the point $v$ can be translated in different ways, the translation which minimises at best the cost function is chosen.

\subsection{Optimisation Strategy}

The purpose of the optimisation strategy is to reach the minimal value of the cost function by iterative removal/addition of simple points or by topology-preserving translations, i.e., to converge to a model topologically equivalent and - as much as possible - geometrically similar to the continuous deformed image $M \circ h$. The selection of simple points to remove/add requires a list $\mathcal{L}$ which contains all simple points of $S$ presenting a positive cost. The cost is defined as the benefit 
to change the label at simple point $v$. More precisely, the modification of $S(v)$ enables to decrease the cost function from the cost $c(v, S, M, h)$ :

$$
c(v, S, M, h)=d(S, M, h)-d\left(S^{\prime}, M, h\right)=\rho(v, S, M, h)-\rho\left(v, S^{\prime}, M, h\right),
$$

where $S^{\prime}$ is the image obtained from $S$ by modifying the value at $v$.

During the dynamical scheme, when modifying a simple point $v$ in $S$ to obtain a new image $S^{\prime}$, there is no need to recompute the whole list $\mathcal{L}$ since $(i)$ $c\left(v^{\prime}, S, M, h\right)=c\left(v^{\prime}, S^{\prime}, M, h\right)$ for all voxels $v^{\prime} \neq v$, and $(i i)$ simple property of points can only be modified in the 26-neighbourhood of $v$. Consequently, the algorithm proceeds as follows until $\mathcal{L}$ is empty. The point of highest cost, denoted $v_{0}$, is removed from the list. The label of $S$ at $v_{0}$ is then modified. This may change the simple points which are in the 26-neighbourhood of $v_{0}$ : points which were not simple (resp. simple) and which become simple (resp. non-simple) must be added if they have a positive cost (resp. removed) in (resp. from) $\mathcal{L}$.

When $\rho(v, S, M, h)=0$, the voxel $v$ belongs to the correct CC. A cost $\rho(v, S, M, h)>0$ can result from the fact that $h(v)$ is at the interface of objects ( $h$ being a continuous field) or from topological constraints. However, it may also result from the convergence of the method to a local minimum. To deal with this issue, we check for all voxels $v$ verifying $\rho(v, S, M, h)>0$ if it is possible to translate $v$ to reduce the cost function without topology modification. It may happen that a translation generates new simple points in the neighbourhood of the involved points, enabling to keep deforming the current image $S$ by "classical" simple point modification.

In order to avoid convergence onto local minima (resulting from geometrical or topological constraints) which can appear with large displacements, the deformation is performed in a "smooth" way by considering $N+1$ intermediate deformation fields computed from $h$, namely $h^{(0)}, h^{(1)}, \ldots, h^{(N)}$ such that:

$$
\left\{\begin{array}{l}
h^{(0)}=I d, h^{(N)}=h \\
\forall j \in[0, N-1], \forall v \in S,\left\|h^{(j+1)}(v)-h^{(j)}(v)\right\|<1(i i)
\end{array}\right.
$$

Constraint (ii) provides a lower bound for $N: N \geq \max _{v \in S}\|h(v)-v\|$. The deformation fields $h^{(i)}(0<i<N)$ are finally defined by:

$$
\forall v \in S, h^{(i)}(v)=v+\frac{i}{N}(h(v)-v) .
$$

The optimisation scheme just described is achieved by considering sequentially $h^{(1)}, h^{(2)}, \ldots, h^{(N)}$. The algorithm is finally described in Alg. 1.

\section{Application: Skull Segmentation from CT Scan Data}

\subsection{Experiments}

One important application of the proposed approach concerns atlas-based segmentation 3. Such methods rely on a binary model $M$ of the structures of 


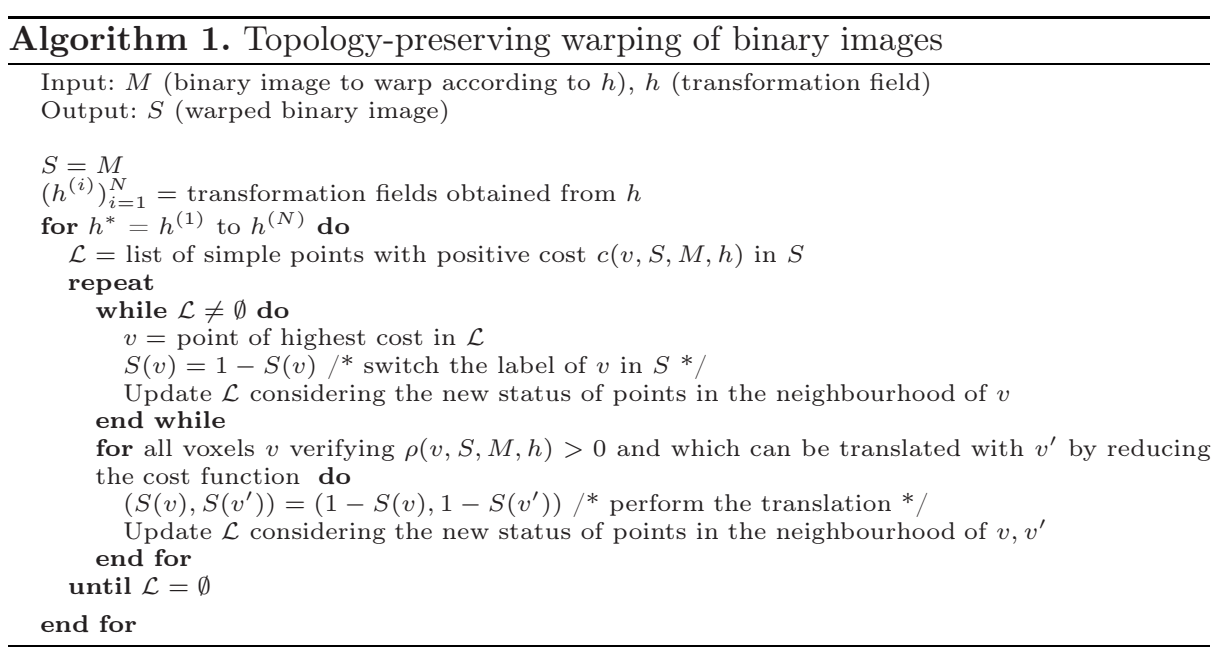

interest which has been obtained from the segmentation of an image $R$. When searching the structures of interest in a new image $I$, the first step consists in estimating a deformation field $h$ by registering $R$ onto $I$. The structures of interest in $I$, denoted $S$, are then obtained by transforming $M$ according to $h$. The binary image topology-preserving deformation is also of great interest since we guarantee that $M$ and $S$ have the same topology. In the sequel, the method is proposed for the segmentation of skull structures from CT-scan data.

The proposed strategy consists in deforming a pre-processed skull template associated to a reference CT image $R$. This template models the parts of the skull which have to be segmented in a geometrically and topologically correct fashion. In particular, it is composed of one connected component, and has no cavity but ten holes corresponding to the foramen magnum, the zygomatic arches, etc. (see Fig. 2). This template is actually the binary image $M$ which has to be warped according to a $3-\mathrm{D}$ deformation field $h$ estimated by registering $R$ onto the $\mathrm{CT}$ image $I$ to be segmented.

\subsection{Results}

The efficiency of the method is not evaluated in terms of segmentation accuracy since it largely depends on the precision of the estimated deformation field. The goal of this experiment is to validate the proposed method $(P M)$ and to show the benefit of the approach with comparison to other interpolation methods, namely, the nearest neighbour interpolation $\left(M_{1}\right)$, and the linear interpolation followed by a thresholding with a threshold of $0.5\left(M_{2}\right)$. These methods are compared from two points of view, a topological one and a geometrical one.

From a topological point of view, the average number of OCCs (see $b_{0}$ in Tab. 1), of holes $\left(b_{1}\right)$, and of cavities $\left(b_{2}=\right.$ number of BCCs -1$)$ are computed for the 15 transported segmentation maps obtained for each method. The proposed method is the only one guaranteeing topology preservation: the topology 

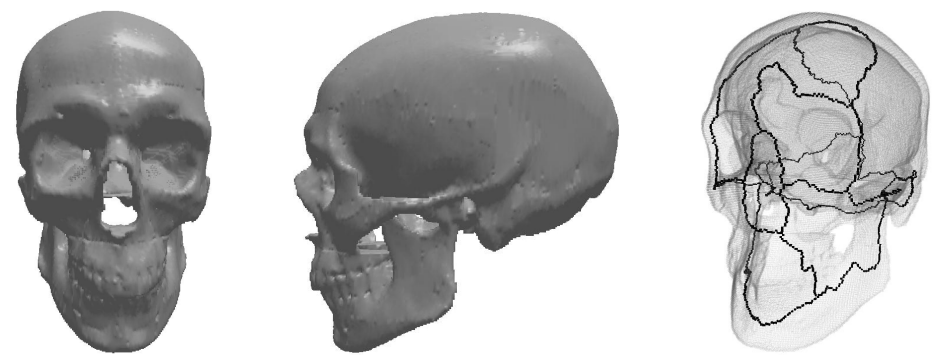

Fig. 2. Skull template used in the proposed application. Left, middle: whole template. Right: template visualised with its topological skeleton. It has to be noticed that this is a partial template: structures such as the vertebrae, for example, are not modelled since their segmentation is not required here.

is strongly altered by methods $M_{1}$ and $M_{2}$ (leading to connected component splitting, hole and cavity generations, etc.). For example, method $M_{1}$ generates on average a number of 150 undesired cavities in the segmentation result. To illustrate this point, Fig. 3 presents a typical result for the proposed method (right) and for the $M_{2}$ method (left).

From a geometrical point of view, the segmentation maps obtained with the proposed approach (Fig. 3, right) is satisfactory since similar to the result obtained with $M_{2}$ (the $M_{1}$ and $M_{2}$ methods provide by construction geometrically correct results). To provide quantitative comparison, the following strategy is used. As $M$ is composed of only one OCC and one BCC, the computation of $\rho(v, S, M, h)$ is possible for each method (to compute $\rho(v, S, M, h)$ for the $M_{1}$ and $M_{2}$ approaches, voxels, for which $S(v)=1$, are associated with the OCC, and the others correspond to the BCC). We observe firstly that the ratios of points for which $\rho(v, S, M, h)=0$ are identical for the three methods $(98.6 \%)$. Results resumed in Tab. 1 provide the ratios of the other points (namely 1.4\%)
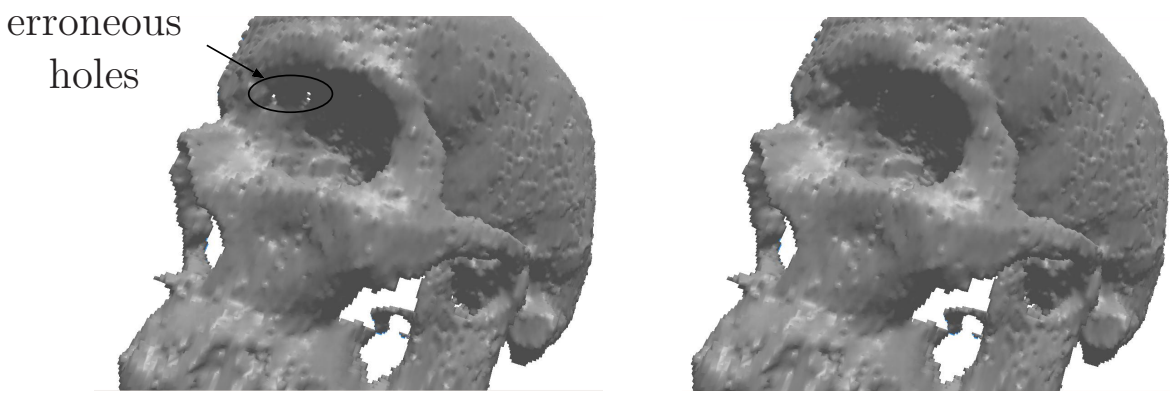

Fig. 3. Segmentation results obtained with method $M_{2}$ (left) and the proposed method (right). The topology has been altered in the left image, but preserved in the right one. Note that the surfaces are visually noisy since the real discrete results are visualised here without mesh generation. 
Table 1. Comparison of the proposed method $(P M)$ from a geometrical $\left(P_{i}, \bar{d}\right.$, and $\left.d_{\max }\right)$ and from a topological $\left(b_{i}\right)$ point of view with the nearest neighbour interpolation $\left(M_{1}\right)$ and the linear interpolation with thresholding $\left(M_{2}\right) . P_{i}$ : ratio (\%) of points $v$ for which $\rho(v, S, M, h)$ is in $](i-1), i] \cdot 25 \cdot 10^{-2} \mathrm{~mm}$ (this ratio is computed without considering voxels for which $\rho(v, S, M, h)$ is equal to 0$) ; \bar{d}$ : mean distance of $\rho(v, S, M, h)$; $d_{\text {max }}$ : maximal value of $\rho(v, S, M, h)$ for the 15 cases; $b_{0}$ (resp. $\left.b_{1}, b_{2}\right)$ : number of object connected components (resp. holes, cavities).

\begin{tabular}{|l|c|c|c|c|c|c|c|c|c|c|}
\hline & $P_{1}$ & $P_{2}$ & $P_{3}$ & $P_{4}$ & $P_{5}$ & $\bar{d}$ & $d_{\max }$ & $b_{0}$ & $b_{1}$ & $b_{2}$ \\
\hline$M_{1}$ & 61.87 & 30.43 & 7.39 & 0.31 & 0.00 & $3.70 .10^{-3}$ & 0.87 & $1.33 \pm 0.59$ & $109 \pm 51.0$ & $150 \pm 71.0$ \\
$M_{2}$ & 62.18 & 37.82 & 0.00 & 0.00 & 0.00 & $3.44 .10^{-3}$ & 0.50 & $1.06 \pm 0.25$ & $24.8 \pm 8.00$ & $7.20 \pm 2.80$ \\
$P M$ & 62.19 & 37.77 & 0.04 & $10^{-3}$ & $10^{-4}$ & $3.44 .10^{-3}$ & 1.06 & $1.00 \pm 0.00$ & $10.0 \pm 0.00$ & $0.00 \pm 0.00$ \\
\hline
\end{tabular}

in terms of distance. The inspection of the results shows that the three methods are relatively similar. Note that the maximal distance is a little bit higher (but still low) for the proposed method. More precisely, on the 15 considered cases, 5 segmentation maps have a unique voxel $v$ whose value $\rho(v, S, M, h)$ is lightly greater than one (the maximal value encountered in the 15 cases for $\rho$ is 1.06). This is due to the fact that the topology preservation induces here geometric constraints. Finally, we can conclude that the geometry is similar for the three methods, but only the proposed approach can correctly handle topology. It has to be noticed that the algorithm has also been tested with objects composed of several CCs. Results are satisfactory but not presented in this paper.

\subsection{Discussion}

We can notice that medical image segmentation methods [4,5] based on topologypreserving deformation of a binary mode 1 have been proposed in the last years. They generally rely on simple algorithmic processes and hypotheses: $(i)$ they use monotonic transformations which either remove or add simple points from/to a model $M$ necessarily surrounding/surrounded by $S,(i i)$ such models are proposed for structures having a non-complex topology or topologically simplified, and (iii) only simple deformation functions (based on grey-level values or distance maps) are considered. The deformation strategy proposed here leads to a segmentation method based on the same concepts but presenting several important improvements: the methods can use non-trivial topological models which evolve in a non-monotonic and topology-preserving fashion under the guidance of complex deformation functions.

\section{Conclusion}

A new method for warping a binary image in a discrete topology preserving fashion according to a continuous topology preserving deformation field has been proposed. Further works will consist in extending this method to label images.

${ }^{1}$ Some methods are based on label models, unfortunately with several approximations 7]8] resulting from still open theoretical problems on label image topology. 
Such extension will require to develop a sound theoretical framework for topological modelling and deformation of such images.

The proposed method has been successfully applied to medical image segmentation. Another perspective is to consider the proposed framework for devising strategies whose behaviour may evolve during the deformation process, for example by performing in parallel segmentation and registration, as proposed in [9].

\section{References}

1. Lehmann, T., Gonner, C., Spitzer, K.: Survey: interpolation methods in medical image processing. IEEE Transactions on Medical Imaging 18(11), 1049-1075 (1999)

2. Bertrand, G., Malandain, G.: A new characterization of three-dimensional simple points. Pattern Recognition Letters 15(2), 169-175 (1994)

3. Dawant, B., Hartmann, S., Thirion, J.P., Maes, F., Vandermeulen, D., Demaerel, P.: Automatic 3-D segmentation of internal structures of the head in MR images using a combination of similarity and free-form deformations: Part I, methodology and validation on normal subjects. IEEE Transactions on Medical Imaging 18(10), 902-916 (1999)

4. Mangin, J.F., Frouin, V., Bloch, I., Régis, J., López-Krahe, J.: From 3D magnetic resonance images to structural representations of the cortex topography using topology preserving deformations. Journal of Mathematical Imaging and Vision 5(4), 297-318 (1995)

5. Dokládal, P., Lohou, C., Perroton, L., Bertrand, G.: Liver blood vessels extraction by a 3-D topological approach. In: Taylor, C., Colchester, A. (eds.) MICCAI 1999. LNCS, vol. 1679, pp. 98-105. Springer, Heidelberg (1999)

6. Passat, N., Ronse, C., Baruthio, J., Armspach, J.P., Bosc, M., Foucher, J.: Using multimodal MR data for segmentation and topology recovery of the cerebral superficial venous tree. In: Bebis, G., Boyle, R., Koracin, D., Parvin, B. (eds.) ISVC 2005. LNCS, vol. 3804, pp. 60-67. Springer, Heidelberg (2005)

7. Bazin, P.L., Pham, D.: Topology-preserving tissue classification of magnetic resonance brain images. IEEE Transactions on Medical Imaging 26(4), 487-496 (2007)

8. Miri, S., Passat, N., Armspach, J.P.: Topologically-based segmentation of brain structures from T1 MRI. In: ISMM 2007, vol. 2, pp. 33-34 (2007)

9. Faisan, S., Passat, N., Noblet, V., Chabrier, R., Armspach, J.P., Meyer, C.: Segmentation of head bones in 3-D CT images from an example. In: ISBI 2008, pp. 81-84 (2008) 